\title{
Aging: toward avoiding the inevitable
}

\author{
Aging is receiving more attention as a risk factor for human disease. With the correct modeling of human \\ heterogeneity and consideration of the environmental factors involved in the aging process, we may be able to delay \\ the onset of human disease.
}

A lthough many would say that aging is a normal part of human biology, age is also the greatest risk factor for a wide variety of chronic disease. Whereas the passing of time cannot be stopped, a growing body of research in model organisms suggests that it may be possible to delay the concurrent decline in health. With this in mind, and with a view to understanding whether these findings in flies, worms and mice can be translated to humans, Nature Medicine-together with the Volkswagen Foundation-organized a conference on aging and age-related disease at the Herrenhausen palace in Hannover, Germany, in September 2015. At the same time, we commissioned the Reviews and Perspectives presented in this Focus issue.

Preclinical data support a 'unifying aging hypothesis', that a common pathway or pathways regulate the aging process and its associated disease indications. Examples of these pathways are covered in the two Reviews in this issue on senescence, page 1424 and metabolism, page 1416, and in a Perspective on proteostasis, page 1406. However, a recurring theme at the September Herrenhausen conference was the heterogeneity of human aging. As noted in a recent report from the World Health Organization (http://apps.who.int/iris/bitstream/ 10665/186463/1/9789240694811_eng.pdf), we do not all age at the same rate with the same prevalence of age-related diseases. Understanding the genetic and/or environmental factors accounting for this heterogeneity, faithfully modeling them in preclinical studies and controlling for them in clinical studies are perhaps the biggest challenges facing the field.

With regard to understanding the genetic underpinnings of aging, inbred model organisms will take us only so far. Interrogating the diversity of the human genome and correlating it with aging phenotypes will be essential. Thanks in part to ever-improving next-generation sequencing technology, these efforts are well under way. In 2007 the Scripps Translational Science Institute launched the Wellderly Study, starting by sequencing a panel of candidate genes associated with aging in cohorts of people and working towards whole-genome coverage. The Longevity Genes Project, which was initiated at Albert Einstein College of Medicine in 2008, also aims to find genes associated with longevity by using a cohort of centenarians, their offspring, and age-matched individuals unrelated to these offspring. More recently, Human Longevity, Inc., a company founded by J. Craig Venter, declared that it is "building the world's most comprehensive database on human genotypes and phenotypes to tackle the diseases associated with aging-related human biological decline." These data sets, which are increasing in terms of their numbers of patients and coverage of the genome, will be invaluable to researchers seeking to unravel the genetic factors that influence human aging.

However, a recent study of over 200 features of the human immune system in identical twins found increasing divergence between identi- cal twins with increasing age, suggesting a marked influence of nonheritable or environmental factors (http://www.cell.com/cell/abstract/ S0092-8674(14)01590-6). Variations in diet, exercise, exposure to toxins and infectious agents, and the composition of the commensal microbiota all exert a profound effect on the well-being of an individual. The challenges lie in teasing apart the contribution of each of these to aging and age-related disease, and in modeling them in preclinical studies.

The latter may be particularly difficult, because although the sequencing methods needed to study the genetics of human aging are now well established, methods for modeling environmental influences on aging in preclinical studies are in their infancy. Consider the typical mouse facility and its sterile, predictable and controlled environment; compare that to the environment throughout a human lifespan. Nonetheless, several ideas for modeling the human environment are being explored, including the domestic dog. Dogs' often-touted nickname 'man's best friend' indicates how closely they share our environment. So, along with their relative genetic homogeneity, which may allow us to distinguish the whether phenotypic heterogeneity can truly be attributed to the environment, dogs may prove to be a good model for gauging how our milieu influences our responses to therapies and/or our health. To this end, scientists at the University of Washington have launched the dog aging project (http:// dogagingproject.com/).

In terms of clinical studies of therapies aimed at extending healthspan, several of the challenges unique to this field are discussed in the Perspective on page 1395 (Viewpoint). But all indications suggest that therapies will be coming. They may be established agents repurposed for treating aging, such as the rapamycin analogs used by Novartis in a proof-of concept trial to boost immune function in the elderly (http:// stm.sciencemag.org/content/6/268/268ra179); see also the Perspective on page 1400 (Dillin). They might also be new agents dedicated to 'drugging' aging. In addition to profiling the genomic and phenotypic aspects of human aging, Human Longevity, Inc. is developing cell therapies aimed at regenerating human tissues. Calico-a company launched by Google-has partnered with the Buck Institute for Research on Aging and the Broad Institute of Massachusetts Institute of Technology to identify therapeutic targets, and with AbbVie to develop drugs to hit these targets. Even with candidate drugs in hand, testing them in clinical trials will require innovation and collaboration with regulatory agencies. The Targeting Aging with Metformin (TAME) study is paving the way toward having healthspan recognized as an indication by regulatory bodies (http://news.sciencemag.org/biology/ 2015/09/feature-man-who-wants-beat-back-aging).

The challenges ahead in dissecting the factors that contribute to aging and age-related disease loom large. With technology, collaboration and innovation, the aging research community will overcome them. 